\title{
Rotulagem nutricional e informações obrigatórias em diferentes marcas de achocolatados em pó
}

\section{Nutrition labeling and mandatory information on different brands of powdered chocolate}

\author{
João Vitor Fonseca Feitoza ${ }^{1}$, Emanuel Neto Alves de Oliveira ${ }^{2}$, Bruno Fonsêca Feitosa ${ }^{3}$, Juvêncio Olegário de Oliveira \\ Neto $^{4}$, Luciano Augusto Marinho Diniz Júnior ${ }^{5}$, Regilane Marques Feitosa ${ }^{6}$
}

\begin{abstract}
Resumo: Achocolatado em pó é um alimento rico em sacarose e outros ingredientes como, por exemplo, o cacau em pó. Ele é comercializado em embalagens rotuladas que apresentam as informações nutricionais do produto, bem como outros itens obrigatórios, sob regras de legislações nacionais específicas. Os rótulos permitem o acesso dos consumidores às informações sobre a composição nutritiva do alimento, possibilitando escolhas mais saudáveis para o consumo. Desta forma, este trabalho objetiva avaliar rótulos de achocolatados em pó comercializados na cidade de Pau dos Ferros, Rio Grande do Norte, verificando suas conformidades frente às legislações em vigência. Foram analisadas 10 marcas de diferentes tipos de achocolatados em pó, comercializadas em diferentes pontos da referida cidade. Verificou-se as informações nutricionais e outras informações obrigatórias utilizando a lista de verificação, segundo dados obrigatórios estabelecidos pela legislação vigente. Observou-se que apenas $60 \%$ das amostras de achocolatados em pó estavam de acordo com as legislações brasileiras para as informações nutricionais e $100 \%$ para as informações obrigatórias. Conclui-se que a maioria das marcas atendem as legislações, no entanto, são necessárias intervenções nas empresas para reparação das não conformidades através dos órgãos fiscalizadores como a Agência Nacional de Vigilância Sanitária e o Ministério da Agricultura, Abastecimento e Pecuária.
\end{abstract}

Palavras-chave: Rótulo; chocolate em pó; legislações.

\begin{abstract}
Powdered chocolate is a sucrose-rich food and other ingredients such as cocoa powder. He is marketed in labeled packagings which present the nutritional information of the product as well as other obligatory items under the rules of specific national legislation. The labels allow consumers access to information on the nutritional composition of the food, enabling healthier choices for consumption. In this way, this work aims to evaluate the labels of chocolate powders commercialized in the city of Pau dos Ferros, Rio Grande do Norteand verify their conformity with the legislation in force. Ten brands of different types of powdered chocolate commercialized in different parts of the city were analyzed. Nutrition information and other mandatory information were verified using the checklist, according to mandatory data established by current legislation. It was observed that only $60 \%$ of the samples of chocolate powders were in accordance with the Brazilian legislation for nutritional information and $100 \%$ for the obligatory information. It is concluded that most brands comply with laws, however, interventions in companies are necessary to repair nonconformities through regulatory agencies such as the National Agency for Sanitary Surveillance and the Ministry of Agriculture, Supply and Livestock.
\end{abstract}

Keywords: Label; Chocolate powder; Legislation.

\footnotetext{
*Autor para correspondência

Recebido para publicação em 23/02/2017; aprovado em 27/06/2017

${ }^{1}$ Discente em Engenharia de Alimentos, Universidade Federal de Campina Grande, Pombal - PB, (83) 998694393, joaovitorlg95@ hotmail.com

${ }^{2}$ Docente do Instituto Federal de Educação, Ciência e Tecnologia do Rio Grande do Norte, Pau dos Ferros - RN, emanuel.oliveira16@ gmail.com

${ }^{3}$ Discente em Engenharia de Alimentos, Universidade Federal de Campina Grande, Pombal - PB, brunofonsecafeitosa@live.com

${ }^{4}$ Discente em Engenharia de Alimentos, Universidade Federal de Campina Grande, Pombal - PB, juvencio_oliveira12@ hotmail.com

${ }^{5}$ Discente em Publicidade e Propaganda, Universidade Potiguar, Natal - RN, oi-luca@ hotmail.com

${ }^{6}$ Discente de Pós-doutorado, Universidade Federal de Campina Grande, Campina Grande - PB, regilanemarques@gmail.com
} 


\section{INTRODUÇÃO}

Diversas embalagens de alimentos possuem rótulos com apresentação da informação nutricional, de acordo com o estabelecido, inicialmente, pela Agência Nacional de Vigilância Sanitária (ANVISA), intervindo por meio da Resolução RDC n 40, de 21 de março de 2001 (BRASIL, 2001), posteriormente revogada pela RDC $\mathrm{n}^{\circ} 360$ (BRASIL, 2003). Isso permite o acesso dos consumidores às informações sobre a composição química dos alimentos, possibilitando escolhas mais saudáveis para o consumo (FERREIRA et al., 2007).

$\mathrm{O}$ acesso fácil à informação sobre o valor nutritivo dos alimentos e suas condições sanitárias provoca polêmica quanto ao grau em que devem ser obrigatoriamente fornecidas aos consumidores. Na elaboração do rótulo de um alimento sempre deve existir uma preocupação quanto à facilidade em compreender as informações (SMITH; ALMEIDAMURADIAN, 2011).

Segundo a Resolução da Diretoria Colegiada (RDC) $n^{\circ}$ 259, de 20 de setembro de 2002 (BRASIL, 2002), o rótulo "é toda inscrição, legenda, imagem ou toda matéria descritiva ou gráfica, escrita, impressa, estampada, gravada, gravada em relevo ou litografada ou colada sobre a embalagem do alimento". Vale ressaltar a importância do rótulo, que é um componente obrigatório nas embalagens, sujeito à multa e apreensão do produto, caso não siga as normas.

Algumas das legislações em vigência sobre rótulos de alimentos são: a RDC n ${ }^{\circ}$ 259, de 20 de setembro de 2002, que aprova o Regulamento Técnico sobre rotulagem de alimentos embalados (BRASIL, 2002), a RDC $\mathrm{n}^{\mathbf{0}} 359$, de 23 de dezembro de 2003, que aprova o Regulamento Técnico de porções de alimentos embalados para fins de rotulagem nutricional (BRASIL, 2003a) e a RDC no 360, de 23 de dezembro de 2003, que aprova o Regulamento Técnico sobre rotulagem nutricional de alimentos embalados, tornando obrigatória a rotulagem nutricional (BRASIL, 2003b).

Frequentemente, encontram-se não conformidades em muitos produtos alimentícios. Como exemplos, Smith e Almeida-Muradian (2011) analisaram 52 rótulos de 13 categorias de alimentos e identificaram no mínimo um tipo de não conformidade em $80,8 \%$ dos rótulos, frente às legislações vigentes. Feitosa et al. (2016a), ao estudar 6 rótulos de diferentes marcas de biscoitos recheados sabor chocolate, identificaram que $16,67 \%$ não informavam o lote e a data de validade dos produtos, ficando claro o descumprimento da legislação. Fonseca et al. (2016) analisou 35 critérios de rotulagem em 7 marcas de biscoitos de chocolate recheados e identificou que 11 critérios apresentavam não conformes com a legislação.

Vários estudos relatam a dificuldade do consumidor em compreender as informações disponibilizadas nos rótulos dos alimentos por não serem claras e concluem que os consumidores não conhecem a rotulagem nutricional e, ainda, alguns não demonstram interesse pela verificação desses dados (CÂMARA et al., 2008; SMITH, ALMEIDAMURADIAN, 2011).

É de grande importância a identificação dos itens da legislação, os quais necessitam de um maior aprimoramento. $\mathrm{O}$ setor de rotulagem carece de estudos para disponibilizar melhor as informações em adequação às necessidades dos consumidores (SMITH; ALMEIDA-MURADIAN, 2011).
Os rótulos possuem informações básicas, dentre elas, a lista de ingredientes, quantidade do alimento, data de fabricação, validade, lote, as próprias informações nutricionais, modo de conservação, dados das empresas envolvidas na produção e comercialização, entre outras. Estas informações devem ser apresentadas de forma clara e objetiva. Também é necessária a indicação da presença de componentes que sejam específicos de um determinado grupo de pessoas, como, por exemplo: hipertensos, diabéticos, celíacos e alérgicos (MOURA et al., 2009; FEITOSA et al., 2016a; FONSECA et al., 2016).

Os achocolatados são alimentos consumidos por pessoas de todas as idades e podem ser encontrados em todo o mundo. As suas características sensoriais e nutricionais, assim como, sua conveniência e praticidade, fazem com que o produto seja bem aceito pelos consumidores. Como esses produtos vêm sendo amplamente comercializados, muitas indústrias têm produzido e aumentado à variedade com preços competitivos. No entanto, o processamento, os ingredientes $\mathrm{e}$ as concentrações utilizadas não são os mesmos, levando a uma variação nas propriedades nutricionais. Em sua apresentação mais simples, o achocolatado contém cerca de $70 \%$ de sacarose ou de outros açúcares e cerca de $30 \%$ de cacau em pó (EDUARDO; LANNES, 2004).

Devido ao alto consumo desse produto no Brasil e a escassez de trabalhos que relatem a adequação das rotulagens frente às legislações, este trabalho objetiva avaliar rótulos de achocolatados em pó comercializados na cidade de Pau dos Ferros Rio Grande do Norte verificando suas conformidades frente às legislações em vigência.

\section{MATERIAL E MÉTODOS}

A pesquisa exploratória foi realizada no mercado local varejista da cidade de Pau dos Ferros (RN) onde foram visitados 10 pontos comerciais. Foram analisadas 10 diferentes marcas de achocolatados em pó (peso líquido de $400 \mathrm{~g}$ ), comercializadas em mercados, mercearias e mercadinhos.

Analisou-se os rótulos das embalagens dos produtos selecionados através de listas de verificação, tendo como base a RDC $n^{\circ} 259$ (BRASIL, 2002), RDC $\mathrm{n}^{\circ} 359$ (BRASIL, 2003a), RDC n 360 (BRASIL, 2003b) e a Lei ${ }^{\circ} 10.674$, de 16 de maio de 2003 (BRASIL, 2003c), que obriga os produtos alimentícios comercializados a informar sobre a presença de glúten, como medida preventiva e de controle da doença celíaca.

A respeito dos produtos verificaram-se as informações nutricionais quanto: porção $(\mathrm{g})$, valor energético (Kcal), carboidratos $(\mathrm{g})$, proteínas $(\mathrm{g})$, gorduras totais $(\mathrm{g})$, gorduras saturadas $(\mathrm{g})$, gorduras trans $(\mathrm{g})$, fibra alimentar $(\mathrm{g})$ e sódio (mg).

Em relação às outras informações obrigatórias nos rótulos, verificou-se: denominação de venda do alimento, lista de ingredientes, medida caseira, conteúdo líquido, identificação da origem, identificação do lote, prazo de validade, conservação do produto, ausência de glúten e nome do país de origem.

\section{RESULTADOS E DISCUSSÃO}

Observa-se na Tabela 1 os resultados para as informações nutricionais das diferentes marcas de achocolatados em pó comercializados na cidade de Pau dos Ferros (RN). 
Diante dos resultados, $80 \%$ das marcas apresentam todas as informações nutricionais exigidas pelas legislações. Apenas $10 \%$ não apresentaram as informações de proteínas, gorduras totais e sódio. $20 \%$ das marcas não apresentaram os valores de gorduras saturadas, gorduras trans e fibra alimentar, estando assim, em desacordo com as RDC $n^{\circ} 359$ (BRASIL, 2003a) e 360 (BRASIL, 2003b).

A informação nutricional é de suma importância para o consumidor, pois é necessário saber o que está adquirindo e consumindo. A maioria das empresas cumprem com a função de informar sobre a composição nutricional dos achocolatados em pó, com informações claras e objetivas, sendo facilmente compreendidas pelos consumidores.

Feitosa et al. (2016a) e Alexandre et al. (2010), ao estudarem diferentes marcas de biscoitos recheados sabor chocolate e rótulos de diferentes marcas de leite em pó integral, respectivamente, também observaram que nem todas as marcas analisadas estavam em conformidade com as legislações vigentes, demonstrando que os erros de rotulagem abrangem várias categorias de alimentos, confirmando o estudo de Smith e Almeida-Muradian (2011).

Porção é a quantidade média do alimento que deveria ser consumida por pessoas sadias, maiores de 36 meses, em cada ocasião de consumo, com a finalidade de promover uma alimentação saudável (BRASIL, 2003b). O valor energético pode ser definido como as quantidades dos nutrientes (em gramas) maioritariamente usados pelo corpo humano, através dos alimentos, para a obtenção de energia - proteínas, lipídeos e glicídeos (SANTOS, 2010). No que se refere às informações porção (g) e valor energético (Kcal), 100\% das marcas as apresentaram, demonstrando atender a este ponto como preconiza a legislação vigente (BRASIL, 2003b).

Tabela 1. Informações nutricionais dos achocolatado em pó comercializados na cidade de Pau dos Ferros, Rio Grande do Norte.

\begin{tabular}{|c|c|c|c|c|c|c|c|c|c|c|}
\hline \multirow{2}{*}{ Informação Nutricional } & \multicolumn{10}{|c|}{ Marcas } \\
\hline & $\mathbf{A}$ & $\mathbf{B}$ & $\mathbf{C}$ & D & $\mathbf{E}$ & $\mathbf{F}$ & $\mathbf{G}$ & $\mathbf{H}$ & $\mathbf{I}$ & $\mathbf{J}$ \\
\hline Porção (g) & 20 & 20 & 10 & 20 & 20 & 12 & 20 & 20 & 20 & 25 \\
\hline Valor Energético (Kcal) & 74 & 80 & 37 & 78 & 73 & 31 & 77 & 78 & 79 & 100 \\
\hline Carboidratos $(\mathrm{g})$ & 17 & 19 & 8,1 & 18 & 18 & 7,8 & 17 & 18 & 19 & 23 \\
\hline Proteínas $(\mathrm{g})$ & 0,7 & 0 & 0,6 & 0,6 & 0 & 0 & 0 & 0,5 & NCT & $<1$ \\
\hline Gorduras Totais $(\mathrm{g})$ & 0,5 & 0 & 0 & 0 & 0 & 0 & 0,4 & 0,5 & NCT & 0 \\
\hline Gorduras Saturadas (g) & 0 & 0 & 0 & 0 & 0 & 0 & 0,2 & NCT & NCT & 0 \\
\hline Gorduras Trans $(\mathrm{g})$ & 0 & 0 & 0 & 0 & 0 & 0 & 0 & NCT & $\mathrm{NCT}$ & 0 \\
\hline Fibra Alimentar (g) & 1,1 & 0 & 0,7 & 0,6 & 0 & 0 & 0,6 & $\mathrm{NCT}$ & $\mathrm{NCT}$ & $<1$ \\
\hline Sódio (mg) & 12 & 28 & 25 & 0 & 26 & 30 & 50 & 71 & $\mathrm{NCT}$ & 30 \\
\hline
\end{tabular}

NCT: Não consta.

Segundo a RDC no 360, carboidratos, hidratos de carbono ou glicídios são todos os mono, di e polissacarídeos, incluídos os polióis presentes nos alimentos, que são digeridos, absorvidos e metabolizados pelo ser humano (BRASIL, 2003b). Das marcas estudadas 100\% apresentaram informações referentes à quantidade deste macronutriente. É importante ressaltar que, segundo Feitosa et al. (2016a), o teor de carboidratos é exemplo de informação útil para diversos profissionais que trabalham na área de controle da alimentação.

Proteínas são polímeros de aminoácidos ou compostos que contém polímeros de aminoácidos (BRASIL, 2003b). Verifica-se que $90 \%$ das marcas apresentaram estes valores, com exceção da marca I, sendo assim a mesma está em não conformidade com a legislação. A marca $\mathrm{H}$ apresenta o valor de $0,5 \mathrm{~g}$, segundo a legislação deveria conter "zero", "0" ou "não contém", pois é considerado não significativo. Magalhães et al. (2016) e Feitosa et al. (2016a) encontraram os valores de proteínas em $100 \%$ das marcas de biscoitos do tipo recheado sabor chocolate, em ambos os trabalhos.

Gorduras ou lipídeos são substâncias de origem vegetal ou animal, insolúveis em água, formadas de triglicerídeos (com ou sem duplas ligações) e pequenas quantidades de não glicerídeos, principalmente fosfolipídeos (BRASIL, 2003b). A RDC no 360 (BRASIL, 2003b) ressalta que "será declarado como "zero", "0" ou "não contém" quando a quantidade de gorduras totais (menor ou igual a $0,5 \mathrm{~g}$ ), gorduras saturadas (menor ou igual a $0,2 \mathrm{~g}$ ) e gorduras trans (menor ou igual a $0,2 \mathrm{~g}$ ) atendem a condição de quantidades não significativas e nenhum outro tipo de gordura seja declarado com quantidades superiores a zero".
Para esses macronutrientes, $40 \%$ das marcas apresentam no mínimo, um tipo de irregularidade. Para gorduras totais, as marcas A, G, e H apresentam valores menores ou igual 0,5 g, quantidade considerada não significativa pela legislação e na marca I não foi encontrado nenhuma informação. Para gorduras saturadas, a marca $\mathrm{G}$ apresentou valor de $0,2 \mathrm{~g}$. Nas marcas H e I não foram encontradas as informações. Para gorduras trans, as marcas $\mathrm{H}$ e I novamente não atenderam a legislação.

As fibras alimentares são componentes importantes, responsáveis por conferir características funcionais ao alimento (FEITOSA et al., 2016a). Observa-se que $80 \%$ das marcas informaram os valores, estando as marcas $\mathrm{H}$ e I em não conformidade com a legislação. Fonseca et al. (2016) encontrou essa informação em $100 \%$ das marcas de biscoitos de chocolate recheados analisados.

O sódio é um mineral essencial, pois atua na manutenção da pressão arterial, além de ajudar em vários processos fisiológicos. Porém, o consumo em excesso contribui para o desenvolvimento da hipertensão (PERTSCHY, 2010). Em relação a esse micronutriente, apenas a marca I não informou a quantidade, estando $90 \%$ das marcas em conformidade.

Observa-se na Tabela 2 os resultados obtidos para as informações obrigatórias avaliadas nos rótulos de achocolatado em pó.

Verifica-se que $100 \%$ das marcas analisadas apresentaram nos rótulos todas as informações obrigatórias exigidas pela legislação brasileira através da RDC $\mathrm{n}^{\circ} 259$ (BRASIL, 2002) e a Lei $n^{\circ} 10.674$ (BRASIL, 2003c) que estabelecem as informações obrigatórias nos rótulos de 
alimentos embalados e obriga os produtos alimentícios comercializados a informar sobre a presença de glúten, como medida preventiva e de controle da doença celíaca, respectivamente.

A denominação de venda do alimento é o nome específico e não genérico que indica a verdadeira natureza e as características do alimento (BRASIL, 2002). Santos et al. (2011) verificou que $100 \%$ dos rótulos de leite em pó integral, comercializados na cidade de Campina Grande, Paraíba estavam conformes em relação a esta informação.

Tabela 2. Informações obrigatórias nos rótulos dos achocolatados em pó comercializados na cidade de Pau dos Ferros, Rio Grande do Norte.

\begin{tabular}{|c|c|c|c|c|c|c|c|c|c|c|}
\hline \multirow{2}{*}{ Informações obrigatórias } & \multicolumn{10}{|c|}{ Marcas } \\
\hline & $\mathbf{A}$ & $\mathbf{B}$ & $\mathbf{C}$ & $\mathbf{D}$ & $\mathbf{E}$ & $\mathbf{F}$ & $\mathbf{G}$ & $\mathbf{H}$ & $\mathbf{I}$ & $\mathbf{J}$ \\
\hline Denominação de Venda do Alimento & CT & CT & CT & CT & CT & CT & CT & CT & $\mathrm{CT}$ & CT \\
\hline Lista de Ingredientes & CT & CT & CT & $\mathrm{CT}$ & $\mathrm{CT}$ & CT & CT & CT & CT & CT \\
\hline Medida Caseira & CT & CT & CT & CT & $\mathrm{CT}$ & CT & CT & CT & CT & CT \\
\hline Conteúdo Líquido (g) & CT & CT & CT & CT & CT & CT & CT & CT & CT & CT \\
\hline Identificação da Origem & CT & $\mathrm{CT}$ & CT & CT & CT & CT & CT & CT & CT & $\mathrm{CT}$ \\
\hline Identificação do Lote & CT & CT & CT & $\mathrm{CT}$ & $\mathrm{CT}$ & CT & CT & CT & $\mathrm{CT}$ & $\mathrm{CT}$ \\
\hline Prazo de Validade & CT & CT & CT & CT & CT & CT & CT & CT & CT & CT \\
\hline Conservação do Produto & CT & CT & CT & CT & CT & CT & CT & CT & CT & CT \\
\hline Ausência de Glúten & CT & CT & CT & CT & CT & CT & CT & CT & CT & CT \\
\hline Nome do País de Origem & CT & CT & CT & CT & CT & CT & CT & CT & CT & CT \\
\hline
\end{tabular}

CT: Consta.

Para a lista de ingredientes, a legislação (BRASIL, 2002) exige a presença no rótulo, devendo vir precedido da expressão "ingrediente:" ou "ing.:" em ordem crescente de quantidade, com os aditivos citados e as suas respectivas funções. Santos et al. (2011), avaliando rótulos de leite em pó integral comercializados na cidade de Campina Grande - PB, observou a lista de ingredientes em $80 \%$ das amostras.

A medida caseira é comumente um utensílio utilizado pelo consumidor para medir alimentos de acordo com a porção descrita no rótulo da embalagem (BRASIL, 2003a). Feitosa et al. (2016b) não encontrou essa informação em 6 rótulos diferentes de iogurtes, considerando 9 analisados.

O conteúdo líquido da embalagem é uma exigência da legislação devendo está precedido das expressões: "PESO LÍQUIDO”, “CONTEÚDO LÍQUIDO”, "PESO LÍQ.”, “Peso Líquido" ou "Peso Líq.", de acordo com as normas exigidas pelo Instituto Nacional de Metrologia, Normalização e Qualidade Industrial (Inmetro), Portaria $\mathrm{n}^{\mathrm{o}} 157$, de 19 de agosto de 2002 (INMETRO, 2002). Costa (2014), avaliando rótulos de embalagens de leites comercializados na região metropolitana de Fortaleza, Ceará, verificou a presença da informação da medida caseira em apenas $58 \%$ dos rótulos.

A identificação de origem, bem como o nome do país, define onde o alimento foi produzido ou, tendo sido elaborado em mais de um país, onde recebeu o último processo substancial de transformação. O lote é o conjunto de produtos de um mesmo tipo, processados pelo mesmo fabricante ou fracionador, em um espaço de tempo determinado, sob condições essencialmente iguais (BRASIL, 2002). O prazo de validade estima basicamente o tempo limite de consumo do alimento. Feitosa et al. (2016a) encontrou não conformidade em $16,67 \%$ dos rótulos analisados quanto a identificação do lote e o prazo de validade.

Apresentar a informação sobre como conservar o produto é uma necessidade para a conservação das características nutricionais do alimento (FEITOSA et al., 2016a). Fonseca et al. (2016) encontrou não conformidade em $14,28 \%$ dos rótulos analisados.

A lei $n^{\circ} 10.674$, de 16 de maio de 2003 (BRASIL, 2003c), preconiza a identificação do produto quanto à presença, ou não, do glúten e objetiva advertir, prevenir e tentar controlar a doença celíaca, que afeta boa parte da população brasileira (BRASIL, 2003c). Yamashita e Carrijo (2014) também verificaram a presença dessa informação em $100 \%$ das amostras, avaliando a rotulagem de patês de diferentes marcas.

\section{CONCLUSÕES}

No comércio de achocolatados em pó na cidade de Pau dos Ferros, Rio Grande do Norte apenas 60\% das amostras estão de acordo com as legislações brasileiras para as informações nutricionais e apresentam $100 \%$ para as informações obrigatórias. Ainda é necessário intervenções nas empresas para a reparação das não conformidades através dos órgãos fiscalizadores como a Agência Nacional de Vigilância Sanitária (ANVISA) e Ministério da Agricultura (MAPA).

\section{REFERÊNCIAS}

ALEXANDRE, A.; AQUINO, A.; OLIVEIRA, E.; FROEHLICH, A. Avaliação da rotulagem de leite em pó integral comercializado em Maceió - AL. In: V Congresso de Pesquisa e Inovação da Rede Norte Nordeste de Educação Tecnológica (CONNEPI), Maceió, 2010.

BRASIL. Ministério da Saúde. Agência Nacional de Vigilância Sanitária - ANVISA. RDC n 40, de 21 de março de 2001. Aprovar o Regulamento Técnico para Rotulagem nutricional obrigatória de alimentos e bebidas embalados.

BRASIL. Ministério da Saúde. Agência Nacional de Vigilância Sanitária - ANVISA. Resolução RDC no 259, de 20 de setembro de 2002. Aprova o Regulamento Técnico sobre Rotulagem de Alimentos Embalados.

BRASIL. Ministério da Saúde. Agência Nacional de Vigilância Sanitária - ANVISA. Resolução RDC n 359, de 23 de dezembro de 2003a. Aprova o Regulamento Técnico de 
porções de alimentos embalados para fins de rotulagem nutricional.

BRASIL. Ministério da Saúde. Agência Nacional de Vigilância Sanitária - ANVISA. Resolução RDC n 360, de 23 de dezembro de 2003b. Aprova o Regulamento Técnico sobre Rotulagem Nutricional de Alimentos Embalados.

BRASIL. Ministério da Saúde. Agência Nacional de Vigilância Sanitária. Lei n ${ }^{\circ} 10.674$, de 16 de maio de 2003c. Obriga a que os produtos alimentícios comercializados informem sobre a presença de glúten, como medida preventiva e de controle da doença celíaca.

CÂMARA, M. C. C.; MARINHO, C. L. C.; GUILAM, M. C.; BRAGA, A. M. C. B. A produção acadêmica sobre a rotulagem de alimentos no Brasil. Revista Panamericana de la Salud Pública, v.23, n.1, p.52-8, 2008.

COSTA, O. A. Avaliação de rótulos de embalagens de leites comercializados na região metropolitana de Fortaleza, Ceará. Revista de Nutrição e Vigilância em Saúde, v.1, n.1, p.18-26, 2014.

EDUARDO, M. F.; LANNES, S. C. S. Achocolatados: análise química. Revista Brasileira de Ciências Farmacêuticas, v.40, n.3, p.405-412, 2004.

FEITOSA, B. F.; OLIVEIRA NETO, J. O.; OLIVEIRA, E. N. A.; FEITOZA, J. V. F.; REGILANE, M. F.; DINIZ JUNIOR, L. A. M. Avaliação da rotulagem de diferentes marcas de biscoitos recheados sabor chocolate comercializados em Pau dos Ferros - RN. Revista A Barriguda, Campina Grande, v.6, n.2, p.230-241, 2016 a.

FEITOSA, B. F.; MESQUITA, J. H. A.; LACERDA, C. G. S.; OLIVEIRA, E. N. A.; SOUZA, R. L. A.; OLIVEIRA, S.N. Avaliação dos rótulos de diferentes marcas de iogurte comercializados na cidade de Pau dos Ferros - RN. In: II Simpósio de Leite e Derivados de Pernambuco, UAG/UFRPE, Garanhuns, 2016b.

FONSECA, J. V. S.; ANDRADE, M. L. FEITOZA, J. V. F.; NOGUEIRA, L. P S.; SILVA, G. V. A.; CAVALCANTI, M. T. Avaliação de rótulos de biscoitos de chocolate recheados comercializados no município de Pombal - PB. Bananeiras: UFPB/ENAG, 2016, p. 398-402.

FERREIRA, M. W.; BRESSAN, M. C.; SOUZA, X. R.; VIEIRA, J. O.; FARIA, P. B.; ANDRADE, P. L. Efeito dos métodos de cocção sobre a composição química e perfil lipídico de filés de tilápia do Nilo (Oreochromis niloticus Linnaeus 1757). Ciência e Agrotecnologia, Lavras, v.31, n.3, p.798-803, 2007.

INMETRO. Instituto Nacional de Metrologia, Qualidade e Tecnologia. Regulamentos Técnicos. Portaria no 157, de 19 de agosto de 2002. Estabelecer a forma de expressar a indicação quantitativa do conteúdo líquido dos produtos prémedidos.

MAGALHÃES, B. N. T.; MENEZES, S. M.; FONSECA, M. T. S.; GOUVEIA, D. S. Avaliação da informação nutricional contida nos rótulos de biscoitos do tipo recheado sabor chocolate. In: Congresso Nacional de Pesquisa e Ensino em Ciências, Campina Grande, 2016.

MOURA, N. C.; CANNIATTI-BRAZACA, S. G.; SILVA, A. G. Elaboração de rótulo nutricional de pães de forma com adição de diferentes concentrações de linhaça (Linum usitatissimum). Alimentação e Nutrição, v.20, n.1, p.149-155, 2009.

PERTSCHY, P. Comparação do teor de sódio em alimentos convencionais, light e diet pela rotulagem dos produto. Trabalho de Conclusão de Curso (Bacharelado em Nutrição) Universidade Estadual do Centro Oeste, Guarapuava, 2010.

SANTOS, D. C.; OLIVEIRA, E. N. A.; MARTINS, J. N. Avaliação da rotulagem de leite em pó integral comercializado na cidade de Campina Grande - PB. Higiene Alimentar, v. 25, n. 194/195, mar./abr. 2011.

SANTOS, R. C. O valor energético dos alimentos. Exemplo de uma determinação experimental, usando calorimetria de combustão. Química Nova, São Paulo, v.33, n.1, p.220-224, 2010.

SMITH, A. C. L.; ALMEIDA-MURADIAN, L. B. Rotulagem de alimentos: avaliação da conformidade frente à legislação e propostas para a sua melhoria. Revista do Instituto Adolfo Lutz, São Paulo, v.70, n.4, p.463-72, 2011.

YAMASHITA, A. S.; CARRIJO, K. F. Avaliação da rotulagem de patês de diferentes marcas produzidos em indústrias com serviço de inspeção sanitária oficial e comercializados no município de Uberlândia, Minas Gerais, Brasil. Enciclopédia Biosfera, Centro Científico Conhecer, Goiânia, v. 10, n. 19; p.271-283, 2014. 